\title{
Extraction of Soil Moisture Change Involved in Soybean Yield by Similarity Evaluation Encompassing Time Series Data
}

\author{
Kurumi Higashiyamaa, ${ }^{\mathrm{a},}$, Ryo Nishide ${ }^{\mathrm{c}}$, Takenao Ohkawaa, \\ Yuya Chonan ${ }^{\mathrm{b}}$, Satoshi Hayashi ${ }^{\mathrm{b}}$, Takuji Nakamura ${ }^{\mathrm{b}}$, Hiroyuki Tsuji ${ }^{\mathrm{b}}$, Noriyuki Murakami ${ }^{\mathrm{b}}$, Seiichi Ozawa ${ }^{\mathrm{a}}$ \\ ${ }^{a}$ Kobe University, Kobe, 657-8501, JAPAN \\ bNARO Hokkaido Agricultural Research Center, Sapporo, 062-8555, JAPAN \\ ${ }^{\mathrm{c} S h i g a ~ U n i v e r s i t y, ~ H i k o n e, ~ 522-8522, ~ J A P A N ~}$ \\ *Corresponding Author: kurumi@ cs25.scitec.kobe-u.ac.jp
}

\begin{abstract}
Our research group aims to analyze and grasp the factors for high and low yielding by using the cultivation data surrounding soybeans. Soil moisture is known to affect the growth of soybean significantly among the cultivation data, but the knowledge to directly improve the yield has not been obtained. In this study, by focusing on time series changes in soil moisture, we propose the method to discover what kind of soil moisture environment causes high or low yield. Since the behavior of soil moisture changes greatly depending on the conditions of the soil, we focus on the gas phase ratio including oxygen and estimate it. In order to grasp what time series change of the gas phase ratio affects the yield, we aim to discover cultivated spots that show similar time series change among many spots. We evaluate the similarity of time series change between spots at each growth stage because soybean requires different environments for each stage. When judging the presence or absence of similarity, it cannot be determined in advance which level or more time series change is similar. Therefore, by evaluating various levels of similarity hierarchically and comprehensively, we adopt the level of similarity that is assumed to be heavily involved in the yield. On the other hand, it may be determined by time series change in several stages, not only a single stage, whether the yield is high or low. Thus, we target the whole stage corresponding to the whole of growth to discover time series change involved in high or low yield. This method was applied to actual data of soil moisture. As a result, we discovered knowledge involved in the generally known low yield, and we also discovered knowledge involved in high yield based on the
\end{abstract}

feature of each growth stage.

Keywords: similarity evaluation, frequent itemsets discovery, soybean.

\section{Introduction}

In recent years, there is a need to support technological succession using scientific method and to improve agricultural efficiency since agricultural workers have been decreasing and aging in Japan. To face with this problem, data mining has been applied to analyze a large amount of accumulated data and to discover empirical knowledge in order to maintain stable production. For example, there are studies to increase the yield of tomatoes and strawberries ${ }^{(1,}$ ${ }^{2)}$, which are crops that are easy to manage. On the other hand, there are several attempts to discover useful knowledge for growing crops under various environmental changes such as land use agriculture.

Soybean is known to be causes of high-yield inhibition, such as pestilence, weed, lodging ${ }^{(3)}$, but not many methods can improve the amount of yield and quality. We aim to analyze and grasp the factors for high and low yielding by using the cultivation data surrounding soybeans. Soybean is surrounded by cultivation data such as the environmental, growth and soil moisture data that are accumulated daily. Among these data, soil moisture is known to affect the growth of soybean significantly, and some studies $^{(4,5)}$ focus on it. However, since these studies compare differences of the growth in specific soil moisture environments, it is difficult to directly determine soil moisture environment affects the yield. Therefore, in this study, by focusing on time series changes in soil moisture, we examine 


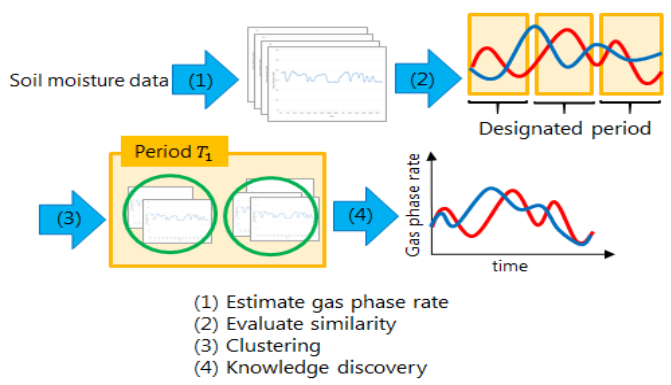

Fig. 1. Framework of proposed method.

the method to discover what kind of soil moisture environment causes high or low yield.

The behavior of soil moisture changes greatly depending on the feature and conditions of the soil. Therefore, even if some cultivated spots have the same value of soil moisture, the growth of crops is different significantly depending on the soil feature. Hence, we focus on the proportion of air in the soil which is said to be more important than soil moisture because it contains oxygen which is needed for the growth. In addition, since soybean requires different environments for each growth stage, it is important to grasp what kind of environment needed for each stage. For these reasons, we attempt to grasp how the proportion of air changes for every designated period corresponding to each growth stage, and discover what kind of time series change of air causes high or low yield. To achieve this, we discover cultivated spots that show similar time series change among many spots for every designated period. At this time, it cannot be determined in advance which level or more time series change can be judged to be similar, so we don't define the level that can be judged to be similar but evaluate comprehensively whether similar or not at various levels. Hence, by evaluating the level of similarity comprehensively, we consider the method to adopt the level of similarity that is assumed to be heavily involved in high or low yield.

On the other hand, it may be determined by time series changes in several periods, not only a single period, whether the amount of yield is high or low. Thus, we target the whole period and focus on cultivated spots with the same level of similarity in each period to discover time series change involved in high or low yield.

\section{Time Series Change Discovery Involved in High or Low Yield Based on Soil Moisture}

\subsection{Overview of the Proposed Method}

In this study, we aim to analyze the factors that cause high or low yield based on survey data obtained for each cultivated spot for experiment. Survey data is roughly divided into soil moisture data and cultivation environment data. Soil moisture data is data that measured the percentage of water in the total soil volume, and it is a time series data that changes with time acquired repeatedly at a certain interval for each cultivated spot. Cultivation environment data is a data that surveyed on soil components and work includes flowering day data, bulk density which is one of the indicators representing the conditions of the soil and fine grain yield data which is the indicator of yield. We can judge whether each spot gives a high or low yield by referring to the fine grain yield. Since soil moisture is important in the growth of soybean, we grasp the characteristic soil moisture environment in either high or low yield spots.

The overview of the proposed method is shown in Fig. 1. At first, as the behavior of soil moisture changes greatly depending on the feature and conditions of the soil, we rather focus on the proportion of air containing oxygen necessary for the growth, which is said to be more important than soil moisture, and use soil moisture and bulk density to estimate the proportion of air, namely, the gas phase ratio (Fig. 1(1)). This gas phase ratio estimated is time series data. Next, in order to grasp what kind of time series change of the estimated gas phase ratio arose and affected high or low yield under what growth conditions, we attempt to discover cultivated spots that show similar time series change among many spots. Soybean requires different environments for each growth stage. Therefore, we evaluate the similarity of time series change between cultivated spots for each designated period corresponding to each growth stage, in order to discover cultivated spots that show similar time series change among many spots (Fig. 1(2)). At this time, it cannot be determined in advance which level or more time series change can be judged to be similar. This is because, depending on the cultivation environment, it may be possible to judge that time series changes are similar if they coincide precisely, on the other hand, to judge that they are similar unless there is a large gap. Therefore, we do not define in advance the level of similarity, but we use hierarchical clustering to evaluate comprehensively whether time series changes are similar or not at various levels (Fig. $1(3))$.

We consider the method to apply for the level of similarity that is assumed to be heavily involved in high or low yield. It may be determined by the interaction of time series changes in several periods, not only a single period, whether the yield is high or low. Thus, we target the whole period 
and focus on cultivated spots with the same level of similarity in each period to discover time series change involved in high and low yield (Fig. 1(4)).

\subsection{Estimation Gas Phase Ratio with Bulk Density}

We estimate the gas phase ratio using soil moisture and bulk density. The behavior of soil moisture changes greatly depending on the feature and condition of the soil. For example, even if the values of soil moisture are the same in several cultivated spots, the growth of crops differs significantly depending on the soil feature, so it may not be possible to analyze uniformly. First, the soil is composed of gas phase (air), liquid phase (moisture) and solid phase (soil particles) and these volume ratios are called gas phase ratio, liquid phase ratio, and solid phase ratio. The various binding of soil particles changes the soil condition, and in particular, the gap size of the particles greatly changes the behavior of moisture. However, since the behavior of air is relatively simple, the oxygen concentration in the gas phase remains almost the same as that in the atmosphere by the gas diffusion. On the other hand, it becomes difficult for the gas diffusion to occur when the gas phase ratio becomes extremely low and lack of oxygen may cause poor growth. Therefore, it is possible to analyze more closely according to the actual cultivation environment by focusing on the gas phase ratio rather than soil moisture (liquid phase ratio).

We calculate the solid phase ratio by bulk density and pure density in order to estimate the gas phase ratio. Bulk density represents the density of a porous substance such as soil and pure density represents the density of only the solid phase portion of the soil. Suppose that bulk density is $b d$ and pure density is $p d$, the solid phase ratio $s$ can be obtained by Equation 1 .

$$
s=\frac{b d}{p d}
$$

Since soil is composed of three phases, assume that soil moisture is $l$ and the gas phase ratio is $g$, Equation 2 holds as follows:

$$
s+l+g=1
$$

Therefore, the gas phase ratio $\mathrm{s}$ can be obtained from Equation 1 and Equation 2. Soil moisture data is time series data, so the gas phase ratio $s$ is also time series data. Note that this gas phase ratio is different from the real measurement data, and is the estimated gas phase ratio.

\subsection{Evaluate Similarity of Time Series Change at Each Designated Period}

We aim to discover cultivated spots that show time se- ries change among many spots in order to grasp what kind of time series change of the estimated gas phase ratio in 2.2 arose and affected high or low yield under a certain growth condition. To perform this, we evaluate the similarity of time series change among all fields. At this time, though the gas phase ratio changes as soybeans grow, it may not be possible to compare the periods to correspond to the same growth stage when evaluating the similarity for the whole period of the data. Moreover, it may be impossible to capture the characteristic time series change at a specific growth stage. In addition, since soybean requires different environments for each growth stage ${ }^{(6)}$, it is important to grasp the kind of environment at each stage. Therefore, we divide the whole process of growth in stages and evaluate the similarity for each period. However, it is necessary to observe the state of growth continuously and to understand the growth stage of soybean in detail. For this reason, it is not easy to obtain information about the growth stage usually. Hence, we divide the whole process of growth into a designated period by using a reference date that clearly indicates the growth stage such as the flowering date to assume a pseudo growth stage, and then, we attempt to grasp the characteristic time series change of gas phase ratio by evaluating the similarity for each period.

Taking into account that the growth of crops is slightly different depending on spots when evaluating the similarity, it is not reasonable to compare time series change at a short time interval as daily. Thereby, we calculate the distance between gas phase ratio data using Dynamic Time Warping $(\mathrm{DTW})^{(7)}$ to evaluate the similarity.

For two time series data, DTW associates one data point in one time series data with multiple data points in the other time series data. Suppose two time series data is $\boldsymbol{x}=$ $x_{1}, x_{2}, \ldots, x_{n_{1}}, \boldsymbol{y}=y_{1}, y_{2}, \ldots, y_{n_{2}}$, where $n_{1}, n_{2}$ is length respectively, The distance $\boldsymbol{x}$ and $\boldsymbol{y}$ is calculated as follows:

1. Construct an $n_{1} \times n_{2}$ matrix $M_{i, j}\left(i=1, \ldots, n_{1}, j=\right.$ $\left.1, \ldots, n_{2}\right)$.

2. Set $\infty$ for all values in the first row and column of the matrix $M$ and $M_{1,1}=0$ as the initial values.

3. Calculate $M_{i, j}(i, j \geq 2)$ by the following Equation 3: $M_{i, j}=\left|x_{i}-y_{j}\right|+\min \left\{M_{i-1, j}, M_{i, j-1}, M_{i-1, j-1}\right\}$ $M_{n_{1}, n_{2}}$ obtained through the above steps is the distance between $\boldsymbol{x}$ and $\boldsymbol{y}$, and the smaller the distance is, the higher the similarity is.

\subsection{Create Data with Similar Time Series Change by Clustering}


We have discovered cultivated spots that show similar time series change among spots based on the similarity in 2.3. At this time, it cannot be determined in advance which level or more time series change can be judged to be similar. This is because, depending on various conditions in cultivation environment, it may be possible to judge that time series changes are similar if they coincide precisely, or on the other, to judge that they are similar unless there is a large gap. Therefore, we cannot define in advance the level of similarity, but we evaluate hierarchically and comprehensively the presence or absence of similarity at various levels of similarity.

We use hierarchical clustering ${ }^{(9)}$ based on the distance matrix $D_{j}$ for a period $T_{j}$ to evaluate the presence or absence of similarity hierarchically and comprehensively at various levels of similarity. $D_{j}$ is a matrix of $n \times n(n$ : The number of spots) and is given as Equation 4.

$$
D_{j}=\left(\begin{array}{cccc}
0 & d_{1,2}^{j} & \cdots & d_{1, n}^{j} \\
d_{2,1}^{j} & 0 & \cdots & d_{2, n}^{j} \\
\vdots & \vdots & \ddots & \vdots \\
d_{n, 1}^{j} & d_{n, 2}^{j} & \cdots & 0
\end{array}\right)
$$

where let period data of a period $T_{j}$ in a spot $i$ is $s_{i, j}=$ $\left\{s_{1, j}, s_{2, j}, \ldots, s_{n . j}\right\}, d_{i, i^{\prime}}^{j}$ is the DTW distance between $s_{i, j}$ and $s_{i^{\prime}, j}$. If $i=i^{\prime}$, period data is same, so the distance equals to 0 .

Hierarchical clustering based on the distance matrix such as Equation 4 forms clusters collected in order from the individual with the smallest distance. Consequently, the similarity of time series change belongs to the cluster is smaller as the size of the cluster is larger. At this point, it is not clear what size of the cluster is important: a small cluster that time series changes coincide precisely may be important, or on the other, a large cluster that they accept a little gap may be important. Therefore, we do not define the size of the cluster in advance but hold the various sizes of clusters hierarchically and comprehensively. Though each cluster is usually exclusive, note that there is an inclusion relation between clusters, as a larger cluster includes a smaller cluster since it is not clear what size of the cluster is important.

We create data with similar time series change $S D$ to hold various sizes of clusters hierarchically and comprehensively. For the number of cluster $m$, a set of clusters $C=\left\{C_{1}, C_{2}, \ldots, C_{m}\right\}$ is represented. Focusing on a cluster $C_{m^{\prime}}$, cluster information is expressed as $D C_{m^{\prime}}=$ $\left\{c f_{1}, c f_{2}, \ldots, c f_{n}\right\}$ using $c f_{i} \in\{0,1\}$ representing whether

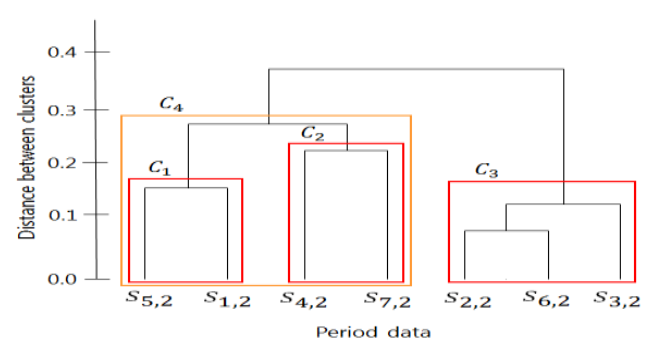

Fig. 2. Example of clustering.

Table 1. Data with similar time series change.

\begin{tabular}{|c|c|c|c|c|}
\hline period data & $D C_{1}$ & $D C_{2}$ & $D C_{3}$ & $D C_{4}$ \\
\hline$S_{1,2}$ & 1 & 0 & 0 & 1 \\
\hline$S_{2,2}$ & 0 & 0 & 1 & 0 \\
\hline$S_{3,2}$ & 0 & 0 & 1 & 0 \\
\hline$S_{4,2}$ & 0 & 1 & 0 & 1 \\
\hline$S_{5,2}$ & 1 & 0 & 0 & 1 \\
\hline$S_{6,2}$ & 0 & 0 & 1 & 0 \\
\hline$S_{7,2}$ & 0 & 1 & 0 & 1 \\
\hline
\end{tabular}

or not period data $s_{i, j}$ belongs to $C_{m^{\prime}}$. By obtaining $D C$ for all clusters and creating data with similar time series change $S D=\left\{D C_{1}, D C_{2}, \ldots, D C_{m}\right\}$, it is possible to hold clusters that have various levels of similarity. For example, Table 1 shows $S D$ when clustering is performed as shown in Fig. 2 when $j=2, n=7, m=4$.

\subsection{Time Series Change Discovery Focusing on Fre- quent Itemsets}

Based on data with similar time series change $S D$ which holds variety of levels of similarity of time series change of the gas phase ratio, we aim to apply for the level of similarity that is heavily involved in the yield and discover time series changes involved in high or low yield. Even though $S D$ is created at each period, time series change should not be investigated in only a single period where high or low yield is involved. The reason is that high or low yield may be determined by the interaction of time series change in several periods. We combine all periods of $S D$ created at each period in all periods and generate the whole period data with similar time series change. For the number of period $k$, the periods are denoted as $T_{1}, \ldots, T_{k}$. Table 2 shows an example of the whole period data with similar time series change. $m_{1}, m_{2}, \ldots, m_{k}$ is the number of clusters for $T_{1}, T_{2}, \ldots, T_{k}$, and $n$ is the number of spots.

We aim to discover time series change involved in high or low yield based on the whole period data with similar time series change. To achieve this, we discover the set of spots with similar time series change that appear 
Table 2. The whole period with similar time series change.

\begin{tabular}{|c|c|c|c|c|c|c|c|c|c|}
\hline & \multicolumn{5}{|c|}{$T_{1}$} & $\cdots$ & \multicolumn{4}{|c|}{$T_{k}$} \\
\hline field & $D C_{1}$ & $D C_{2}$ & $\cdots$ & $D C_{m_{1}}$ & $\cdots$ & $D C_{1}$ & $D C_{2}$ & $\cdots$ & $D C_{m_{k}}$ \\
\hline 1 & 1 & 0 & $\cdots$ & 1 & $\cdots$ & 0 & 1 & $\cdots$ & 1 \\
\hline 2 & 0 & 0 & $\cdots$ & 0 & $\cdots$ & 0 & 0 & $\cdots$ & 0 \\
\hline 3 & 0 & 0 & $\cdots$ & 0 & $\cdots$ & 0 & 0 & $\cdots$ & 0 \\
\hline 4 & 0 & 1 & $\cdots$ & 1 & $\cdots$ & 0 & 1 & $\cdots$ & 1 \\
\hline 5 & 1 & 0 & $\cdots$ & 1 & $\cdots$ & 1 & 0 & $\cdots$ & 1 \\
\hline$\cdots$ & $\cdots$ & $\cdots$ & $\cdots$ & $\cdots$ & $\cdots$ & $\cdots$ & $\cdots$ & $\cdots$ & $\cdots$ \\
\hline$n$ & 0 & 1 & $\cdots$ & 1 & $\cdots$ & 1 & 0 & $\cdots$ & 1 \\
\hline
\end{tabular}

commonly in several periods. This results in frequent itemsets discovery ${ }^{(10)}$ when each spot is regarded as a transaction and each cluster at each period is regarded as an item. In this study, we use Apriori algorithm ${ }^{(11)}$, which can efficiently search and enumerate frequent itemsets from large basket data by using the feature of support. By applying Apriori algorithm for each basket data of high and low yield spots, we discover the set of spots with time series change involved in high or low yields. The basket data $B D$ is a set of transaction data $T D$, and it is represented by $B D=\left\{T D_{1}, T D_{2}, \ldots, T D_{n}\right\}$ for the number of transaction $n$. For the number of item $l$, let $I=\left\{I_{1}, I_{2}, \ldots, I_{l}\right\}$ be a set of item, then the $i$ th transaction data is represented as $T D_{i}=$ $\left\{t_{1}, t_{2}, \ldots, t_{l^{\prime}}\right\}\left(1 \leq j \leq l^{\prime}, t_{j} \subseteq I\right)$. In this study, the cluster $C_{m_{k^{\prime}}}$ is represented as the item $I_{T_{k^{\prime}}} C_{m_{k^{\prime}}}$ at a period $k^{\prime}$, and the basket data $B D$ is created since each cluster at each period is an item.

We discover frequent itemsets by Apriori algorithm based on itemsets $X$ of high or low yield obtained from $B D$. Itemsets $X$ is subset of $I$. Apriori algorithm can efficiently discover frequent itemsets $F$ by using the feature of support support $(X)$. The support $(X)$ represents the ratio of transactions in which itemsets $X$ appears for $n$. Frequent itemsets $F$ is the itemsets that satisfies $\operatorname{support}(\mathrm{X}) \geq \operatorname{minSup}$ for the minimum support minSup. minSup needs to be appropriately defined depending on the number of periods with similar time series change and whether the yield is high or low. This is because, the number of high yield spots may be different from that of low yield spots, and also because if the number of periods in which spots appear commonly increases, it may be difficult to find such spots. Therefore, we set minSup appropriately according to the number of periods for high and low yield, respectively.

By setting minSup appropriately and applying
Apriori to each basket data of high and low yield spots, $H F$ that enumerates high-yielding frequent itemsets and $L F$ that enumerate low-yielding frequent itemsets are obtained. However, $H F$ and $L F$ do not necessarily enumerate frequent itemsets specific to high or low yield since they may enumerate the same frequent itemsets. The reason is that clustering is performed with high and low yield spots mixed together and data with similar time series change is created. Thus, we obtain $H F^{\prime}$ and $L F^{\prime}$ that enumerate frequent itemsets specific to high or low yield according to the following as:

$$
\begin{aligned}
& H F^{\prime}=\left\{F \mid F_{1} \nsubseteq F_{2}\right\} \\
& L F^{\prime}=\left\{F \mid F_{1} \nsupseteq F_{2}\right\}
\end{aligned}
$$

where $F_{1} \in H F, F_{2} \in L F$. Moreover, we set a parameter $\alpha$ which represents the bias toward high or low yield to obtain frequent itemsets that may be heavily involved in high or low yield. A set of high yield spots which have frequent itemsets $F^{\prime}$ is denoted as $F_{H}\left(F^{\prime}\right)$ and a set of low yield spots is denoted as $F_{L}\left(F^{\prime}\right)$. We define $\alpha$ as the following equation. We accept $F^{\prime}$ where $\alpha$ is greater than or equal to the threshold $t h$.

$$
\alpha= \begin{cases}\frac{\left|F_{H}\left(F^{\prime}\right)\right|}{\left|F_{H}\left(F^{\prime}\right)\right|+\left|F_{L}\left(F^{\prime}\right)\right|} & \left(F^{\prime} \in H F^{\prime}\right) \\ \frac{\left|F_{L}\left(F^{\prime}\right)\right|}{\left|F_{H}\left(F^{\prime}\right)\right|+\left|F_{L}\left(F^{\prime}\right)\right|} & \left(F^{\prime} \in L F^{\prime}\right)\end{cases}
$$

\section{Experiment}

\subsection{Dataset}

We verify that knowledge involved in yield can be found by applying the proposed method to actual soil moisture data. Survey data is provided by NARO Central Agricultural Research Center, and this data is collected from cultivated spots in Japan. The data is acquired in 2016, and the cultivated spots targeted for the experiment are 130 spots. By referring to the fine grain yield data in the survey, spots with a yield of $250(\mathrm{~kg} / 10 \mathrm{a})$ or above are classified as high yield spots and less than $250(\mathrm{~kg} / 10 \mathrm{a})$ as low yield spots. Consequently, there are 61 spots for high yield, and 60 spots for low yield. 42 days of soil moisture data are used based on one week before the flowering day, which is a common period for all spots. The number of days in a designated period is 7 days, and the number of periods is $k=6$. As mentioned in Section 2.2, pure density is necessary to estimate the gas phase ratio, though it was not measured in spots for this experiment. However, since pure density $p d$ does not change much with soil, set $p d=2.6$ 
Table 3. Detail setting of minimum support.

\begin{tabular}{|c|c|c|}
\hline $\begin{array}{c}\text { Number of similar } \\
\text { periods }\end{array}$ & $\begin{array}{c}\text { Number of fields } \\
\text { in high yield }\end{array}$ & $\begin{array}{c}\text { Number of fields } \\
\text { in low yield }\end{array}$ \\
\hline 1 & 6 & 7 \\
\hline 2 & 5 & 5 \\
\hline 3 & 5 & 5 \\
\hline
\end{tabular}

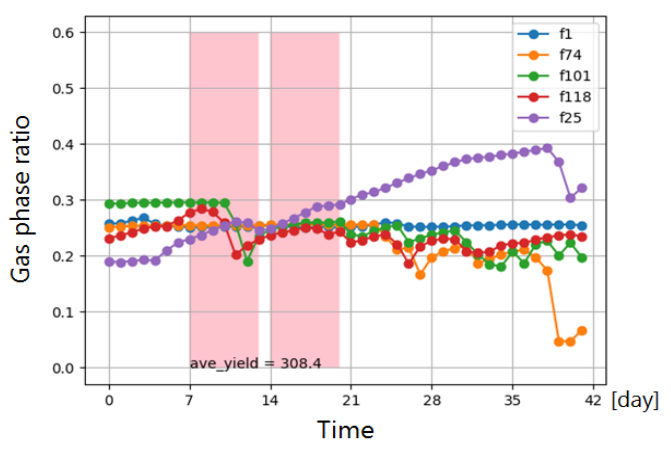

Fig. 3. Example 1 of high yield spots.

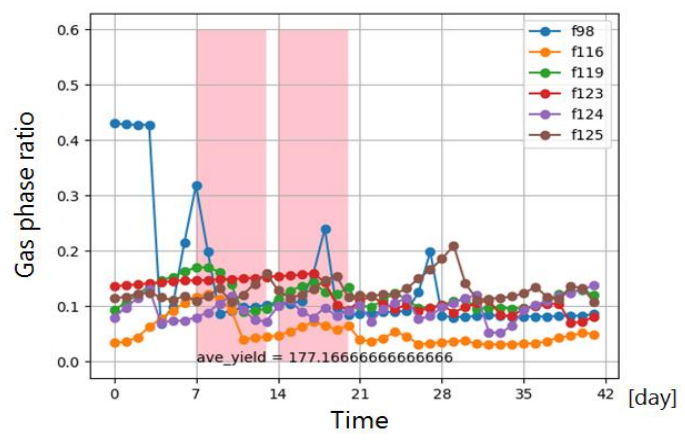

Fig. 4. Example 1 of low yield spots.

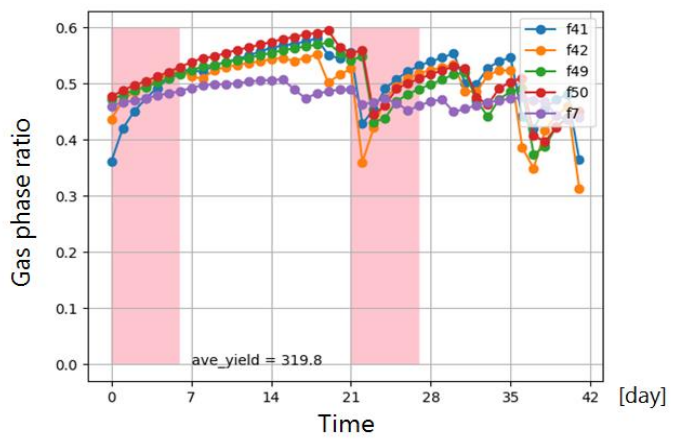

Fig. 5. Example 2 of high yield spots. for all spots. Moreover, since the real gas phase ratio is not measured in spots for this experiment, the validation of the estimated data is not performed for this experiment. Table 3 shows the detail setting of the minimum support described in Section 2.5. The Ward method is used for clustering to create data with similar time series change. If the number of periods with similar time series change is 4 or above, then it is omitted from the experiment because the number of spots is extremely small. The threshold for determining the frequent itemsets to be adopted is $t h=0.7$.

\subsection{Results}

Fig. 3 to 6 show the time series change of the gas phase ratio involved in high or low yield obtained by applying the proposed method. The horizontal axis shows the number of days from one week before the flowering day, and the vertical axis shows the estimated gas phase ratio. The pink area is the period that is assumed to be involved in high or low yield represented by frequent itemsets obtained by the proposed method. Numbers such as $\mathrm{f} 1$ in the legend indicate cultivated spot numbers.

\subsection{Discussion}

We compare Fig. 3 and Fig. 4. In Fig. 4, which shows low yield spots, the estimated gas phase ratio hovers around $10 \%$ in both pink periods. This means that water in the soil is stagnant due to poor drainage or long rain. As a result, it is considered that decreases in water absorption by crops and in transpiration from plants have occurred. Therefore, the factor of low yield is assumed to be the wet damage caused by them. Moreover, two weeks after flowering is the time when pods begin to grow, and the stagnation of water during this period may affect the yield. The cause of water stagnation is assumed to be poor drainage except in the spot f98. In f98, drainage may be good, but it is assumed that rainfall exceeded drainage. On the other hand, in Fig. 4, which shows high yield spots, the estimated gas phase ratio hovers from 20 to $30 \%$. This value indicates that air in the soil is sufficient because it is desirable for the growth of soybean to have the gas phase ratio of $12 \%$ or above. Thus, this can be a feature that leads to high yield. 
Next, we discuss Fig. 5, which shows high yield spots. At first, it is observed that the estimated gas phase ratio hovers around $50 \%$ in the first period. This indicates that the proportion of air in the soil is large and the soil is a bit dry. If the soil is dry before flowering, the crop tends to extend its roots deeply for absorbing water. This enables to absorb water and nutrients efficiently from a wide area, so the crop can absorb water from the soil deeply even if the soil is dry. In the second period, the estimated gas phase ratio drops and then rises. This indicates that the soil was moistened by the heavy rainfall, but dried immediately. As factors of the increase of the gas phase ratio, it is assumed that drainage was good or transpiration from plants was active. It is known among experts that these factors lead to high yield.

Finally, we discuss Fig. 6, which shows low yield spots. In Fig. 6, the estimated gas phase ratio hovers around $10 \%$ in both pink periods. This indicates that it was raining continuously, which causes a disease and has a negative affect such as the lack of nutrients by the loss of transpiration from plants. It is assumed that the wet damage occurred due to these factors and caused low yield.

\section{Conclusions and Future Works}

In this paper, by comprehensively evaluating the similarity of time series change of the estimated gas phase ratio in every designated period, we propose the method to discover soil moisture environment involved in high or low yield. For experiment on the actual soil moisture data, we discovered general knowledge involved in low yield such as wet damage. Moreover, we discovered knowledge involved in high yield based on the feature of each growth period.

The drawbacks of this study are as follows. In the method, it is necessary to integrate environmental factors

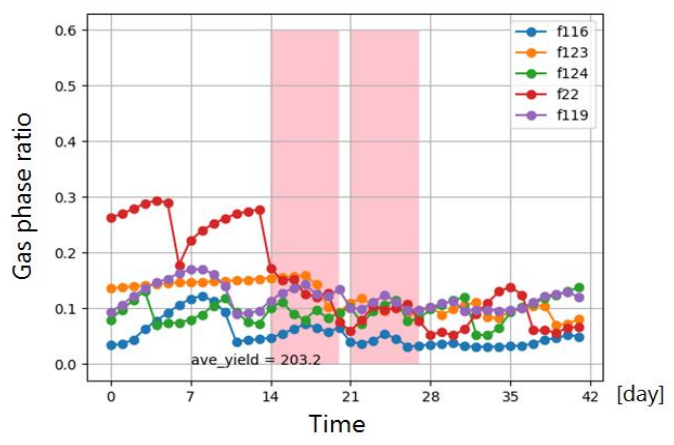

Fig. 6. Example 2 of low yield spots. that affect the condition of the soil. Although soil moisture is important in cultivation, weather factors also affect soil moisture environment. Specifically, precipitation is an environmental factor that may directly affect the soil conditions, so it is necessary to develop a method considering this factor. For the experiment, it is necessary to carefully examine the acquired knowledge. Since the result obtained with the proposed method may be different from the knowledge of the experts, it is uncertain if such results actually affected in high or low yield. Therefore, it is necessary to verify the efficiency together with other environmental factors such as soil components and solar radiation. In addition, it is also necessary to investigate whether the obtained knowledge is also applicable for the following years.

\section{Acknowledgment}

This study was partially supported by Ministry of Agriculture, Forestry and Fisheries: Development of diagnostic methods and countermeasure techniques for overcoming high-yield inhibitory factors.

\section{References}

(1) P. Makelaa, K. Jokinend, M. Kontturic, P. Peltonen-Sainioa, E. Pehua, and S. Somersaloa: "Foliar application of glycinebetaine - a novel product from sugar beet - as an approach to increase tomato yield", Industrial Crops and Products, Vol. 7, Issues 2-3, pp. 139-148, 1998.

(2) B. Z. Yuan, J. Sun, and S. Nishiyama: "Effect of Drip Irrigation on Strawberry Growth and Yield inside a Plastic Greenhouse", Biosystems Engineering, Vol. 87, Issues 2, pp. 237-245, 2004.

(3) Japan Agricultural Development and Extension Association (JADEA), 2012: "Soybean Making for Improvement of Yield and Quality and Stable Production Q \& \&”, http://www.jadea.org/houkokusho/daizu/documents/dai zu-kaitei.pdf, pp. 2-5, Accessed 15 June 2017 (in Japanese).

(4) T. Hirasawa, M. Nakahara, T. Izumi, Y. Iwamoto, K. Ishihara, "Effects of Pre-Flowering Soil Moisture Decits on Dry Matter Production and Ecophysiological Characteristics in Soybean Plants under Well Irrigated Conditions during Grain Filling”, Plant Plod. Sci., Vol. 1, Issues 1, pp.8-17, 1998. 
(5) M. G. Huck, K. Ishihara, C. M. Peterson, T. Ushijima, "Soybean Adaptation to Water Stress at Selected Stages of Growth”, Plant Physiol., Vol. 73, pp.422-427, 1983.

(6) W. R. Fehr, C. E. Caviness, "Stages of Soybean Development", Cooperative Extension Service, Iowa State University, Special Report 80, 1977.

(7) D. J. Berndt, J. Clifford, "Using Dynamic Time Warping to Find Patterns in Time Series", AAAI-94 Workshop on Knowledge Discovery in Databases, KDD-94, pp.359-370, 1994.

(8) Z. Wang, H. Hao, Y. Lu, “A three-phase soil model for simulating stress wave propagation due to blast loading", INTERNATIONAL JOURNAL FOR NUMERICAL AND ANALYTICAL METHODS IN GEOMECHANICS, Vol. 28, Issues 1, pp.33-56, 2004.

(9) C. Aggarwal, "Data Mining: The Textbook", Springer International Publishing, pp.166-172, 2015.

(10) R. Agrawal, T. Imielinski, A. Swami, "Mining Association Rules between Sets of Items in Large Databases", Proceedings of the 1993 ACM SIGMOD international conference on Management of data, pp.207-216, 1993.

(11) R. Agrawal, R. Srikant, "Fast algorithms for mining association rules", Proceedings of the 1994 international conference on very large data bases, pp.487-499, 1994. 